Isabel Aninat, Verónica Figueroa y Ricardo González (editores), El pueblo mapuche en el siglo XXI. Propuestas para un nuevo entendimiento entre culturas en Chile (Santiago: Centro de Estudios Públicos, 2017).

RESEÑA

\title{
DESAFÍOS EN AUTONOMÍA Y RECONOCIMIENTO CONSTITUCIONAL DE LOS PUEBLOS INDÍGENAS
}

\author{
Miriam Henríquez Viñas \\ Universidad Alberto Hurtado
}

L os artículos de los profesores Manuel Antonio Núñez ("Pueblos indígenas y su reconocimiento constitucional. Formas de autonomía no territorial y territorial", 2017) y de Salvador Millaleo ("Los derechos políticos de los pueblos indígenas y la encuesta mapuche del CEP", 2017) se escribieron con una perspectiva constitucional e internacional en el libro El pueblo mapuche en el siglo XXI. Propuestas para un nuevo entendimiento entre culturas en Chile. Este libro fue editado por Isabel Aninat, Verónica Figueroa y Ricardo González, y publicado en 2017 por el Centro de Estudios Públicos (CEP). La obra colectiva recoge una serie de trabajos que interpretan, con un enfoque interdisciplinario, los resultados de las encuestas aplicadas por el CEP al pueblo mapuche en 2006 y 2016, con la finalidad de proponer reflexiones y recomendaciones de política pública indígena.

Los artículos recién mencionados abordan el reconocimiento político y constitucional de los pueblos indígenas y sus derechos colectivos, centrando su análisis en los derechos políticos, temática principal del

Miriam Henríquez Viñas. Abogado por la Universidad Nacional del Comahue, Argentina. Doctora en Ciencias Jurídicas por la Universidad de Santiago de Compostela. Profesora de Derecho Constitucional en la Universidad Alberto Hurtado. Email: mhenriqu@uahurtado.cl. 
constitucionalismo latinoamericano de finales del siglo XX y principios del siglo XXI.

Una de las características materiales de las constituciones del llamado nuevo constitucionalismo latinoamericano es que plantean "la integración de los sectores marginados históricamente, como es el caso de los pueblos indígenas" (Viciano y Martínez 2011, 22). En este ámbito, destaca el amplio reconocimiento de derechos que provienen de las más distintas tradiciones - desde la democrática, hasta el indigenismo, pasando por el socialismo- ideologías y cosmovisiones (Salazar 2013, 356).

En el sentido apuntado, la constitución boliviana de 2009 establece en su artículo 1 el Estado plurinacional; luego garantiza la libre determinación de los pueblos indígenas en el marco de la unidad del Estado, que consiste en su derecho a la autonomía, al autogobierno, a su cultura, al reconocimiento de sus instituciones (artículo 2); propone un amplio catálogo de derechos de los pueblos indígenas (artículo 30 y ss.); establece el pluralismo jurídico (artículo 178); asegura el reconocimiento de la autonomía indígena (artículos 289 y ss.); determina un sistema de jurisdicción indígena sin relación de subordinación con la jurisdicción ordinaria (artículos 179 II, 192 y 410); establece la elección de sus representantes políticos a través de sus formas propias de elección (artículo 211); y propone la creación de un Tribunal Constitucional Plurinacional con presencia de la jurisdicción indígena (artículo 179).

A su vez, la constitución ecuatoriana de 2008 prescribe en el artículo 1 que Ecuador es un Estado intercultural y plurinacional. Luego señala que la nacionalidad ecuatoriana es el vínculo jurídico político de las personas con el Estado, sin perjuicio de su pertenencia a alguna de las nacionalidades indígenas que coexisten en el Ecuador plurinacional (artículo 6 párrafo 2); más adelante consagra las circunscripciones territoriales indígenas o afroecuatorianas, que ejercerán las competencias del gobierno territorial autónomo, y que se regirán por principios de interculturalidad, plurinacionalidad y de acuerdo con los derechos colectivos (artículo 257); y, por último, establece la responsabilidad del Estado de proteger el patrimonio cultural que es configurado por la identidad plurinacional, pluricultural y multiétnica del Ecuador (artículo 380). Por su parte, los derechos colectivos están ampliamente recogidos en los artículos 57, 60 y 171. Esta carta también reconoce la justicia indígena en su artículo 171. 
Por otro lado, las constituciones del modelo democrático constitucional —originario del constitucionalismo de posguerra que acompañó las olas democratizadoras, con impacto en algunos países latinoamericanos, como México, Argentina y Colombia- conforman un modelo complejo, que combina las tradiciones liberal, democrática y social. En tal contexto recogen un amplio catálogo de derechos fundamentales, como libertades, derechos políticos y sociales (Salazar 2013, 347-350), entre ellos, el reconocimiento de los pueblos indígenas y sus derechos.

Así, por ejemplo, la constitución colombiana de 1991 prevé normas de reconocimiento y protección de la diversidad étnica y cultural de la nación colombiana (artículo 7); propone el reconocimiento de la oficialidad, en sus respectivos territorios, de las lenguas y dialectos de los grupos étnicos y la enseñanza bilingüe en estas zonas (artículo 10); garantiza el derecho de los integrantes de los grupos étnicos a una formación que respete y desarrolle su identidad cultural (artículo 68 inciso 5); entrega el derecho a la nacionalidad para los miembros de los pueblos indígenas que comparten territorios fronterizos (artículo 96 n. ${ }^{\circ}$ ); establece la existencia de circunscripciones especiales para la elección de senadores por parte de las comunidades indígenas (artículo 171); determina la existencia de circunscripciones especiales para la elección de miembros a la Cámara de Representantes por parte de las comunidades indígenas (artículo 176); y asegura el derecho de las autoridades de los pueblos indígenas a ejercer funciones jurisdiccionales de conformidad con sus propias normas y procedimientos, dentro de sus territorios (artículo 246).

Por otro lado, la constitución argentina, reformada en 1994, en su artículo 75 inciso 17 reconoce la preexistencia étnica y cultural de los pueblos indígenas argentinos; garantiza el respeto a su identidad y el derecho a una educación bilingüe e intercultural; reconoce la personería jurídica de sus comunidades, y la posesión y propiedad comunitarias de las tierras que tradicionalmente ocupan; regula la entrega de otras aptas y suficientes para el desarrollo humano; y asegura su participación en la gestión referida a sus recursos naturales y a los demás intereses que los afecten.

Las citas a las normas constitucionales expuestas ilustran cómo los aspectos constitucionalizados, ya por la vía de enmiendas o de nuevos textos constitucionales, han ido desde el mínimo reconocimiento de la 
multiculturalidad del Estado hasta el establecimiento de derechos culturales de los pueblos indígenas. De esta forma, las cartas fundamentales latinoamericanas han reconocido:

a) a los pueblos indígenas como pueblos originarios y preexistentes;

b) la propiedad de sus tierras, como recurso material, cultural y soporte político de la existencia de aquéllos;

c) una serie de derechos tales, como los derechos a la identidad y cultura propia, el derecho a conservarla, desarrollarla y transmitirla; a la autodeterminación o autogobierno según sus usos y costumbres; a la administración privilegiada sobre los recursos naturales existentes en sus territorios; a la religión ancestral como factor de identificación étnico cultural; a tener formas propias de organización social; a la participación en la política local y nacional mediante la elección de sus propios representantes; a ser consultados cada vez que se promuevan medidas legislativas y administrativas susceptibles de afectarlos; y a adaptar la justicia a los sistemas indígenas utilizando sus usos y costumbres, entre otros derechos (Henríquez 2005, 129).

Es posible entonces constatar la gran heterogeneidad normativa en cuanto al alcance, contenido y formulación de las normas constitucionales que reconocen a los pueblos indígenas en las constituciones, lo que hace difícil identificar un modelo único replicable en esta materia (Aguilar et al. 2011, 60). Empero, el factor común en las cartas del nuevo constitucionalismo latinoamericano y en las cartas latinoamericanas del modelo democrático constitucional es que han abordado - con distintas cualidades, énfasis e intensidades - la cuestión indígena en un intento "por recuperar a los excluidos de entre los excluidos" (Gargare1la 2013, 22).

No obstante, la constitución política de Chile ha permanecido ajena a esta tendencia latinoamericana y no ha recogido la pluralidad cultural, ni ha aceptado la multiculturalidad del Estado, ni ha reconocido la existencia de los pueblos indígenas, y tampoco sus derechos o el deber del Estado de respetarlos. Esta afirmación no supone desconocer los adelantos que se han procurado en el tratamiento de la cuestión indígena, en los niveles legal, internacional y de programas gubernamentales.

Aunque han sido varios los intentos por incorporar el reconocimiento de los pueblos indígenas y sus derechos colectivos en el texto de la actual constitución chilena, ninguno de ellos ha logrado el consenso 
político necesario. Permanece, en consecuencia, pendiente. Las razones que se han expuesto para justificar los rechazos pueden resumirse en:

a) no es posible concebir un Estado con más de una nación;

b) no es viable que coexistan en un Estado unitario ordenamientos jurídicos distintos;

c) no es razonable, desde la dogmática de los derechos fundamentales, atribuir derechos a entes colectivos;

d) cualquier reconocimiento de derechos colectivos a grupos afectaría el principio de igualdad ante la ley; y

e) todo reconocimiento podría ser interpretado como la afirmación implícita de un derecho a la autodeterminación política de esos pueblos, con lo que se crearía un Estado dentro de otro. Este último sentido, Román se lo atribuye a los parlamentarios opositores a las distintas propuestas de modificación (Román 2014, 145).

Cabe apuntar que la referencia a lo "pendiente" es a un reconocimiento constitucional explícito, toda vez que por vía del artículo 5 inciso segundo de la Constitución se incorporan internamente los tratados, declaraciones y estándares del derecho internacional de los derechos humanos, plasmados, por un lado, en el Convenio 169 de la Organización Internacional del Trabajo, sobre Pueblos Indígenas y Tribales en Países Independientes (1989), en la Declaración de las Naciones Unidas sobre los Derechos de los Pueblos Indígenas (2007) y en la Declaración Americana sobre los Derechos de los Pueblos Indígenas (2016), y, por otro, en la jurisprudencia de la Corte Interamericana de Derechos $\mathrm{Hu}-$ manos.

Recapitulando, el primer proyecto de reforma a la Constitución sobre la temática fue presentado por el Presidente Patricio Aylwin (Boletín 513-07) el 7 de enero de 1991. En virtud de este proyecto se incorporaba al artículo 1 de la Constitución un inciso final que rezaba: "El Estado velará por una adecuada protección jurídica y el desarrollo de los pueblos indígenas que integran la nación chilena". Asimismo, modificaba el artículo 19 n. ${ }^{\circ} 22$, en el sentido de permitir el establecimiento de franquicias tributarias a favor de las comunidades indígenas, y reformaba el artículo 62, en cuanto a establecer como iniciativa exclusiva del Presidente de la República la promoción de leyes tendientes a la protección jurídica y beneficios o franquicias para el desarrollo de los pueblos indígenas. Fue la expresión "pueblos indígenas", contemplada 
en las disposiciones reformadas, la que llevó a conflictos, puesto que por "pueblo indígena" se entendió un ente colectivo autónomo ubicado entre los individuos y el Estado, con facultad de autodeterminación. Como consecuencia del rechazo de la expresión "pueblos indígenas", el Gobierno de la época decidió sustituir dicha mención simplemente por "indígenas"; empero, este cambio no bastó para lograr los acuerdos políticos necesarios para la aprobación de la reforma constitucional, la que se archivó el 9 de julio de 1997.

En mayo de 1999, el Ejecutivo solicitó el desarchivo del proyecto mencionado y su nueva tramitación por el Congreso Nacional. La Comisión de Derechos Humanos, Nacionalidad y Ciudadanía de la Cámara de Diputados acordó incorporar un inciso final al artículo 1 de la Constitución, cuyo texto decía: "El Estado reconoce a los pueblos indígenas, los cuales integran la nación chilena. La ley promoverá el respeto y desarrollo de sus culturas, lenguas, organización social y costumbres, y garantizará a sus integrantes mecanismos de participación en los asuntos que les competen, en iguales términos que a los demás sectores o grupos que conforman la nación chilena". Luego del segundo informe de la Comisión de Derechos Humanos, la Cámara de Diputados votó el proyecto de reforma constitucional, que resultó rechazado por no reunir el quórum exigido.

También en el año 1999, los diputados Huenchumilla, Elgueta, García, Tuma, Hernández, Ojeda, Luksic, Bustos, Ceroni y Letelier presentaron una moción de reforma constitucional, que reconocía a los pueblos indígenas y les otorgaba participación política en el Estado (Boletín 2360-07, del 6 de julio de 1999). Esta propuesta no alcanzó tramitación en la Cámara de Diputados.

En el año 2000, la Concertación de Partidos por la Democracia, a través de los senadores Bitar, Hamilton, Silva y Viera-Gallo, presentó un proyecto de reforma constitucional que consideraba la cuestión indígena (Boletín 2534-07, del 6 de julio de 2000). El proyecto presentado constaba de tres artículos. El primero indicaba agregar como inciso final al artículo 1 el siguiente: "El Estado velará por la adecuada protección jurídica y el desarrollo de los pueblos indígenas que integran la nación chilena"; agregar como inciso final al número 22 del artículo 19 el siguiente: "La ley podrá también establecer los beneficios o franquicias determinadas a favor de las comunidades indígenas"; y agregar al 
artículo 62, como número 7 nuevo, el siguiente: "Establecer sistemas de protección jurídica y beneficios o franquicias para el desarrollo de los pueblos indígenas".

Luego de un arduo debate en el seno de la Comisión de Constitución, Legislación, Justicia y Reglamento del Senado, ella aprobó un reconocimiento implícito a los pueblos indígenas a través de la mención constitucional de los mismos como objeto de tutela del Estado, con un marcado acento en la indivisibilidad de la nación chilena. El texto de la proposición se determinó de esta manera: intercalar en el artículo 1 de la carta fundamental, como incisos tercero y cuarto, nuevos, los siguientes: "La nación chilena es indivisible. El Estado reconoce la diversidad de origen de los chilenos que forman parte de la nación y declara su especial preocupación por las poblaciones indígenas originarias, a las cuales garantiza su derecho a fortalecer los rasgos esenciales de su identidad".

Tal moción fue discutida, pero no alcanzó las mayorías necesarias para su aprobación. De este modo, la reforma constitucional de 2005 no recogió la cuestión indígena, quedando nuevamente diferida la respuesta del Estado de Chile en el nivel constitucional.

Una nueva propuesta de reforma constitucional tuvo su origen en un proyecto que refundió la moción de los senadores Espina, Allamand, Cantero, García y Romero, presentado a tramitación en septiembre de 2007 (Boletín 5522-07), y en el mensaje de la Presidenta de la República, Michelle Bachelet, de noviembre del mismo año, Boletín 5324-07, y actualmente en tramitación.

El texto del proyecto plantea reemplazar el artículo 4 de la Constitución por el siguiente: "La nación chilena es una, indivisible y multicultural. Se reconoce la existencia de los pueblos indígenas que habitan el territorio de Chile y el derecho de sus comunidades, organizaciones e integrantes a conservar, fortalecer y desarrollar su identidad, cultura, idiomas, instituciones y tradiciones y a participar en la vida económica, social, política y cultural del país en la forma que establece el orden jurídico nacional. Los pueblos indígenas podrán organizar su vida de acuerdo a sus costumbres, siempre que ello no contravenga la Constitución y las leyes". Asimismo, intercalar en el actual artículo 5 el inciso primero: "Chile es una república democrática"; intercalar en el 19 n. ${ }^{\text {o }}$, entre las palabras "mujeres" y "son", la siguiente frase: "cualquiera sea 
su origen étnico o racial"; y agregar en el artículo 19 n. ${ }^{\circ} 24$ inciso final: "La ley debe proteger la propiedad sobre las tierras de las personas y comunidades indígenas y sus derechos de aprovechamiento de aguas conforme a lo establecido en la Constitución y las leyes".

Otro proyecto de enmienda constitucional, iniciado por moción del 11 de julio del 2012, por los diputados Arenas, Auth, Chahin, De Urresti, Jaramillo, Meza, Monckeberg, Teillier, Tuma y Vargas (Boletín 8438-07), y actualmente en tramitación, plantea: i) reconocer la existencia de los pueblos indígenas, estableciendo que estos son titulares de derechos colectivos garantizados por la Constitución, uno de los cuales es el derecho a la participación y representación política; y ii) reformar las disposiciones sobre la composición de la Cámara de Diputados y del Senado, generando diez cargos de diputados y cuatro de senadores, estableciendo que ellos serán llenados por representantes de los distintos pueblos indígenas que en cada caso se indica, en base a una elección que se realizará simultáneamente con la elección parlamentaria. En estos casos, los electores serán las personas pertenecientes a cada etnia, en base a un Registro Electoral Indígena a cargo del Servicio Electoral, cuya regulación queda en manos de una ley orgánica constitucional. Estos diputados y senadores tendrán las mismas atribuciones, derechos y obligaciones del resto de los parlamentarios, con dos excepciones: la primera es que en el caso de los senadores su mandato durará cuatro años y la segunda es que, en caso de vacancia, los reemplazantes deberán definirse por elecciones complementarias. También se postula modificar las normas que regulan la conformación de los consejos regionales y de los concejos municipales, creando cupos supernumerarios que permitan asegurar a representantes indígenas en regiones. Esta misma institución debe ser creada en las comunas en las que exista más del 25 por ciento de población indígena censada.

Finalmente, cabe destacar el proceso de cambio constitucional llevado a cabo por el segundo gobierno de la Presidenta Michelle Bachelet, el que contempló de forma inédita un proceso constituyente indígena, específico y diferenciado del general. Éste consistió en recoger los temas que los pueblos indígenas de Chile consideran deben ser previstos en una nueva carta fundamental (proceso realizado entre agosto y diciembre de 2016) y someter estos resultados en 2017 a un proceso de consulta constituyente indígena, con el fin de elaborar los planteamientos que serán incluidos en un próximo proyecto de nueva constitución. 
Analizado el panorama constitucional latinoamericano y chileno, cabe destacar que los artículos de los profesores Manuel Núñez y Salvador Millaleo ponen de relieve esta falta de reconocimiento de los pueblos indígenas y de sus derechos colectivos en la Constitución Política de la República de Chile, así como la conexión que existe entre la demanda de los movimientos indígenas por dicho reconocimiento y el estado de la opinión pública, según los resultados de la encuesta mapuche del CEP 2016. Ambos trabajos enfatizan el conflicto indígena y resaltan que el Estado chileno no ha encontrado soluciones eficaces al mismo o señalan que las propuestas institucionales han sido insuficientes. Los trabajos coinciden en que una respuesta posible a dicha situación de conflicto es la constitucionalización de la multiculturalidad y de los derechos colectivos políticos; sin embargo, previenen al unísono que las respuestas constitucionales que se planteen deben exceder de una mera retórica constitucional. En cierta medida, los autores encuentran parte de la solución en la constitucionalización de los derechos colectivos políticos, basados en la autodeterminación de los pueblos indígenas, fundamentalmente en las autonomías no territoriales y territoriales, y más particularmente en los cupos reservados para indígenas en alguna de las ramas del Congreso Nacional.

El punto de partida expuesto en su trabajo por Manuel Núñez, profesor de la Universidad Católica de Valparaíso, es que los ordenamientos políticos deben reconocer la existencia de una pluralidad de culturas y, luego, de una pluralidad de pueblos, es decir, el multiculturalismo. Ante la ausencia de un reconocimiento constitucional formal en Chile de los pueblos indígenas, el autor propone una serie de vías alternativas, tales como:

a) la constitucionalización a través del ius gentium y formas pacticias;

b) la constitucionalización a través del reconocimiento judicial; y

c) la constitucionalización a través de la reforma constitucional o del ejercicio del poder constituyente originario.

Respecto a la primera alternativa planteada, Núñez expone que "el legado de los tratados abre una vía para una nueva forma de acuerdos o pactos constitutivos que, fuera del léxico internacionalista y ya dentro del constitucional, ofrecen a largo plazo oportunidades de reconocimiento, reconciliación y mecanismos incrementales y regionales de acomodo que bien pueden desafiar en ventajas a los instrumentos constitucionales unilaterales de reconocimiento" (Núñez 2017, 122). 
La segunda alternativa, posiblemente la menos simbólica - a juicio de Núñez-, consiste en "integrar, mediante procesos judiciales de interpretación, a las comunidades indígenas y sus derechos en el lenguaje de las constituciones vigentes" (Núñez 2017, 123). Esto se lograría mediante la recepción de los avances experimentados en el derecho internacional de los derechos humanos por la jurisprudencia del Tribunal Constitucional y de la Corte Suprema, los que, por ejemplo, han reconocido el derecho a la consulta a los pueblos indígenas.

Por su parte, la tercera vía, sobre la que se ha dado cuenta en la primera parte de esta reseña, supone una reforma constitucional o la dictación de una nueva constitución. El contenido de los ámbitos de la regulación constitucional depende — en opinión de Núñez- de tres variables jurídicas: "La textura de las reglas constitucionales, los estándares internacionales y la experiencia comparada" (Núñez 2017, 124). Sin embargo, existe un mínimo que el autor estima necesario regular: "La autonomía en dos tipos o modalidades que son complementarias. La primera de ellas es la autonomía territorial y se refiere a las formas de descentralización o autogobierno dentro del país. La segunda es la llamada autonomía no territorial y alude a una variedad multiforme de técnicas de atribución de poder político a grupos determinados, donde la transferencia de poder no va asociada al control de un territorio específico" (Núñez 2017, 126). Núñez destaca que en el caso chileno parece aconsejable dirigir la mirada a las formas de autonomía no territorial, atendidos:

a) el carácter mixto de la población;

b) el predominio de los territorios compartidos con población no indígena; $\mathrm{y}$

c) la concentración de la población indígena en las ciudades.

Apoyado en la encuesta mapuche CEP 2016, el articulista concluye que es necesario "descartar soluciones etnocentristas de autogobierno exclusivamente indígena” (Núñez 2017, 127).

Manuel Núñez presenta las distintas formas de autonomía no territorial y sugiere su preferencia por algunas de ellas:

a) el derecho de organización política, expresado a través de partidos o de organizaciones consuetudinarias propias reconocidas por el Estado; b) la capacidad de crear un derecho propio 
(usualmente consuetudinario) coercible frente a las instituciones estatales (como costumbre vinculante al menos en disputas entre miembros de una misma etnia) o propias (mediante la llamada justicia indígena); c) la capacidad de decidir sobre asuntos de naturaleza cultural, como la educación, la lengua o la religión; d) el derecho de participación en las instancias decisorias de políticas estatales nacionales o locales; e) la capacidad para hacerse representar ante asambleas o cuerpos colegiados con poderes de decisión, y f) la autoridad para opinar a través de cuerpos consultivos locales o nacionales. (Núñez 2017, 127)

Fundado en los resultados de la encuesta mapuche CEP 2016, el profesor Núñez se inclina por la opción de crear cupos reservados para indígenas, puntualmente en la Cámara de Diputados, y en número proporcional a la población indígena nacional, asunto que requeriría de ciertos ajustes constitucionales y legales en la mecánica electoral.

En lo que respecta a la autonomía territorial, Núñez expone la viabilidad de profundizar y avanzar tomando como punto de partida las instituciones conocidas. En tal sentido - y en su opinión-, la reforma municipal puede pasar por la reformulación de las comunas o por la puesta en valor de las unidades vecinales. Lo anterior podría ser reforzado con otros procesos de participación general, como las consultas y los plebiscitos comunales. Tales propuestas requerirían de cambios legales y habilitaciones constitucionales.

Por su parte, el artículo del profesor de la Universidad de Chile Salvador Millaleo busca destacar "cómo pueden los derechos colectivos políticos aportar a resolver la insuficiencia institucional del Estado de Chile para tratar con las conflictividades" (Millaleo 2017, 158).

Salvador Millaleo da cuenta pormenorizadamente de las características de los derechos colectivos, y los sitúa en el multiculturalismo democrático. El principal derecho colectivo político es —a su juiciola autodeterminación; esto es, el derecho de los pueblos indígenas para determinar su propio futuro y sus propias formas de gobierno (Millaleo 2017, 176). En este contexto, el derecho a la libre determinación "se ejerce a través de autonomías y formas de autogobierno, pero incluye también derechos de participación y el derecho al propio sistema jurídico" (Millaleo 2017, 177).

El profesor Millaleo explica que 
la autonomía indígena, en los países que la han incorporado, consiste en un poder legalmente fundado de un pueblo para ejercer funciones públicas, independientemente de otras fuentes de autoridad estatal, pero sujeto a un orden jurídico y a autoridades estatales comunes. La autonomía normalmente consistirá en la transferencia de poderes de gobierno a entidades organizadas de los pueblos indígenas sobre una base territorial, en cuanto esos pueblos están normalmente vinculados espiritualmente a una territorialidad determinable. Dentro de las autonomías territoriales pueden distinguirse entre regímenes autonómicos y el reconocimiento a entidades regionales indígenas. (Millaleo 2017, 177)

También define a las autonomías no territoriales como "la transferencia de poderes de gobierno a entidades indígenas sin una base territorial, sino bajo la orientación de un principio personal, de manera que dichas entidades pueden detentar y ejercer derechos correspondientes a los pueblos indígenas" (Millaleo 2017, 178).

Salvador Millaleo concluye que el reconocimiento de los derechos colectivos políticos de los pueblos indígenas requiere del reconocimiento de su libre determinación interna. En un sentido coincidente con el profesor Núñez, sugiere para Chile más bien la representación especial y formas de autonomías no territoriales. Respecto a la representación especial, señala la conveniencia de realizarla a través del sistema de escaños reservados antes que el de cuotas (Millaleo 2017, 190).

Finaliza expresando que ojalá la "combinación de la autonomía territorial, la no territorial y la representación especial como contenido normativo concreto del deseado reconocimiento constitucional pueda asentar las bases institucionales para la superación del conflicto" (Millaleo 2017, 190).

Para concluir, los desafíos expuestos en ambos artículos, en orden a la constitucionalización del reconocimiento de los pueblos indígenas y sus derechos, son:

a) atender a un cambio constitucional integral y no fragmentado de la Constitución;

b) comprender que de la riqueza de las reglas constitucionales no se sigue necesariamente un aumento en el disfrute de los derechos de los pueblos indígenas, debiendo considerarse normas subconstitucionales que aseguren una eficacia material; 
c) distinguir lo puramente constitucional de lo legal, considerando los niveles de acuerdo político; y

d) apoyar toda decisión en datos empíricos y estadísticos objetivos para tomar el pulso a las intenciones ciudadanas, en cuyo marco destacan la valía y el aporte de la encuesta mapuche que, en dos ocasiones —2006 y 2016 — ha aplicado el Centro de Estudios Públicos.

\section{REFERENCIAS}

Aguilar, G., S. Lafosse, H. Rojas \& R. Steward. 2011. Justicia constitucional y modelos de reconocimiento de los pueblos indígenas. México: Porrúa.

Aninat I., V. Figueroa \& R. González, eds. 2017. El pueblo mapuche en el siglo XXI. Propuestas para un nuevo entendimiento entre culturas en Chile Santiago: Centro de Estudios Públicos.

Centro de Estudios Públicos. 2006. Encuesta "Los mapuches rurales y urbanos hoy". https:/cepchile.cl/cep/site/artic/20160304/asocfile/20160304094057/encCEP_ may2006_mapuche.pdf

_. 2016. Encuesta "Los mapuches rurales y urbanos hoy". Marzo-mayo. https:// cepchile.cl/cep/site/artic/20160607/asocfile/20160607195127/encuestacep_ mapuche_marzo_mayo2016.pdf

Gargarella, R. 2013. "Nuevo constitucionalismo latinoamericano y derechos indígenas. Una breve introducción". Boletín Onteaiken 15: 22-32.

Henríquez, M. 2005. "Los pueblos indígenas y su reconocimiento constitucional pendiente”. En Reforma Constitucional, coordinado por F. Zúñiga, 127-145. Santiago: LexisNexis.

Millaleo, S. 2017. "Los derechos políticos de los pueblos indígenas y la encuesta mapuche del CEP”. En Aninat, Figueroa \& González 2017, 155-196.

Núñez, M. A. 2017. "Pueblos indígenas y su reconocimiento constitucional. Formas de autonomía no territorial y territorial”. En Aninat, Figueroa \& González 2017, 111-154.

Román, C. 2014. "Reconocimiento constitucional de los pueblos originarios en Chile". Revista de Derecho Público (número especial marzo): 137-147.

Salazar, P. 2013. "El nuevo constitucionalismo latinoamericano (una perspectiva crítica)". En El constitucionalismo contemporáneo. Homenaje a Jorge Carpizo, coordinado por D. Valadés \& L. González, 345-387. México: UNAM.

Viciano, R. \& R. Martínez. 2011. "El nuevo constitucionalismo latinoamericano: fundamentos para una construcción doctrinal". Revista General de Derecho Público Comparado 9: 1-24. EP 
